\title{
Determination of $\left|V_{u d}\right|$ from nuclear mirror transitions
}

\author{
O. Naviliat-Cuncic ${ }^{1}$ and N. Severijns ${ }^{2}$ \\ ${ }^{1}$ LPC-Caen, ENSICAEN, Université de Caen Basse-Normandie, CNRS/IN2P3-ENSI, Caen, France \\ ${ }^{2}$ Instituut voor Kern- en Stralingsfysica, Katholieke Universiteit Leuven, B-3001 Leuven, Belgium
}

(Dated: October 26, 2018)

\begin{abstract}
The $V_{u d}$ element of the Cabibbo-Kobayashi-Maskawa quark mixing matrix has traditionally been determined from the analysis of data in nuclear superallowed $0^{+} \rightarrow 0^{+}$transitions, neutron decay and pion beta decay. We show here that this element can independently be determined from nuclear mirror transitions. The extracted value, $\left|V_{u d}\right|=0.9719 \pm 0.0017$, is at 1.2 combined standard deviations from the value obtained in superallowed $0^{+} \rightarrow 0^{+}$transitions and has a similar precision than the value obtained from neutron decay experiments. We discuss some prospects to improve its precision through experiments in nuclear mirror transitions.
\end{abstract}

PACS numbers: 12.15.Hh; 23.40.-s; 24.80.+y

The unitarity conditions of the Cabibbo-KobayashiMaskawa (CKM) quark mixing matrix [1, 2] provide sensitive means to test the consistency of the three generation standard electroweak model and to search for new physics beyond. A stringent test is obtained from the elements of the first row

$$
V_{u d}^{2}+V_{u s}^{2}+V_{u b}^{2}=1
$$

where $V_{u j}$ denotes the amplitude of the quark mass eigenstate $j$ into the quark weak eigenstate $d^{\prime}$. The accuracy in the verification of this condition is largely due to the dominant value and error of the $V_{u d}$ element which is obtained from weak decay processes involving the lightest quarks.

Three traditional sources to determine $\left|V_{u d}\right|$ from experiments have been considered during the past decades, namely, nuclear superallowed $0^{+} \rightarrow 0^{+}$pure Fermi transitions, neutron decay and pion beta decay, and these have regularly been reviewed $[\underline{3}, 4,[5]$.

The partial half-lives of nine nuclear superallowed $0^{+} \rightarrow 0^{+}$transitions have been studied in great detail [6, 7]. Measurements of lifetimes, masses and branching ratios have reached precisions such that the required inputs for the calculation of the $\mathcal{F} t$ values were obtained at a level of few parts in $10^{-4}$, yielding the value [7]

$$
\left|V_{u d}\right|=0.97418(26) \text { (superallowed } 0^{+} \rightarrow 0^{+} \text {), }
$$

The value of $\left|V_{u d}\right|$ deduced from $0^{+} \rightarrow 0^{+}$transitions is since many years [3] dominated by uncertainties in theoretical corrections. Present experimental activities are oriented toward further reducing these uncertainties by testing the calculations in other transitions [7].

Neutron decay involves both the vector and the axialvector interactions so that the determination of $\left|V_{u d}\right|$ from neutron decay data, although free of nuclear structure corrections, requires the analysis of at least two observables. The most precise determinations have so far been obtained by combining the neutron lifetime with the beta asymmetry parameter. The first determination of
$\left|V_{u d}\right|$ using only neutron decay data 8] yielded the value $\left|V_{u d}\right|=0.9790(30)$. The present world average recommended value for the neutron lifetime, $\tau_{n}=885.7(8) \mathrm{s}$ [9], combined with the world average value for the beta asymmetry parameter, $A_{n}=-0.1173(13)$ [9], yields

$$
\left|V_{u d}\right|=0.9746(19) \text { (neutron decay). }
$$

The improvement by a factor of about 1.5 over almost two decades shows the difficulty of the associated experiments (see e.g. 10, 11]). Other results have however been reported [11, 12] by taking selected values of the most precise experimental data. Many experimental projects are under way 13 to improve the uncertainties on the neutron lifetime and on several of the correlation parameters.

Finally, the absolute pion beta decay rate provides a clean observable for the determination of $\left|V_{u d}\right|$. The main experimental difficulty arises here from the very weak $\left(10^{-8}\right)$ branching of the beta decay channel. The most recent experimental determination yields [14]

$$
\left|V_{u d}\right|=0.9728(30) \text { (pion decay), }
$$

what is less precise than the value from neutron decay.

We consider here a new source to determine $\left|V_{u d}\right|$, namely, the beta decay transitions between $T=1 / 2$ isospin doublets in mirror nuclei. Such transitions are sometimes called "mirror decays" and similarly to neutron decay -which is the simplest mirror transition- proceed via the vector and axial-vector interactions. The principle to extract $\left|V_{u d}\right|$ from such transitions is then similar to that used in the analysis of neutron decay, except for the corrections associated with the nuclear system. The corrections for the determination of the $\mathcal{F t}$ values in mirror transitions have recently been surveyed 15] and were obtained with sufficient precision for their consideration in the analysis reported here. We use then below the results of this new survey and adopt the definitions and notations given there, unless possible ambiguities require it otherwise. 
The vector part of the corrected statistical decay rate function is given by [15]

$$
\mathcal{F} t \equiv f_{V} t\left(1+\delta_{R}^{\prime}\right)\left(1+\delta_{N S}^{V}-\delta_{C}^{V}\right)
$$

where $f_{V}$ is the uncorrected statistical rate function, $\delta_{R}^{\prime}$ denotes nuclear dependent radiative corrections obtained from QED calculations, $\delta_{N S}^{V}$ are nuclear structure corrections and $\delta_{C}^{V}$ are isospin symmetry breaking corrections for the vector contribution. For mixed Fermi/GamowTeller transitions, $\mathcal{F} t$ is related to $V_{u d}$ by [15]

$$
\mathcal{F} t=\frac{K}{G_{F}^{2} V_{u d}^{2}} \frac{1}{C_{V}^{2}\left|M_{F}^{0}\right|^{2}\left(1+\Delta_{R}^{V}\right)\left(1+f_{A} \rho^{2} / f_{V}\right)}
$$

where $K /(\hbar c)^{6}=2 \pi^{3} \ln 2 \hbar /\left(m_{e} c^{2}\right)^{5}$ and has the value $K /(\hbar c)^{6}=8120.278(4) \times 10^{-10} \mathrm{GeV}^{-4} \mathrm{~s}, G_{F} /(\hbar c)^{3}=$ $1.16637(1) \times 10^{-5} \mathrm{GeV}^{-2}$ is the Fermi constant [9], $C_{V}=$ 1 is the vector coupling constant, $\Delta_{R}^{V}$ is a transitionindependent radiative correction [16], $f_{A}$ is the statistical rate function for the axial-vector part of the interaction, and $\rho$ is the Gamow-Teller to Fermi mixing ratio. This ratio is defined by 15]

$$
\begin{aligned}
\rho & =\frac{C_{A} M_{G T}^{0}}{C_{V} M_{F}^{0}}\left[\frac{\left(1+\delta_{N S}^{A}-\delta_{C}^{A}\right)\left(1+\Delta_{R}^{A}\right)}{\left(1+\delta_{N S}^{V}-\delta_{C}^{V}\right)\left(1+\Delta_{R}^{V}\right)}\right]^{1 / 2} \\
& \approx \frac{C_{A} M_{G T}^{0}}{C_{V} M_{F}^{0}} .
\end{aligned}
$$

where the square root contains the nuclear structure, isospin symmetry breaking and radiative corrections for the vector and axial-vector contributions, $C_{A}$ is the axialvector coupling constant $\left(C_{A} / C_{V} \approx-1.27\right)$ and $M_{F}^{0}$ and $M_{G T}^{0}$ are the isospin symmetry limit values of the Fermi and Gamow-Teller matrix elements, with $\left|M_{F}^{0}\right|^{2}=1$ for the $T_{i}=T_{f}=1 / 2$ mirror transitions.

Using the corrected $\mathcal{F} t$ values from the recent compilation [15] it is possible to extract $\left|V_{u d}\right|$ from Eq.(6) provided another observable, also function of $\rho$, be known with sufficient precision. In the present analysis we consider transitions where the beta-neutrino angular correlation coefficient, $a_{\beta \nu}$, and the beta decay asymmetry parameter, $A_{\beta}$, have been measured in the past. For $\beta^{+}$ mirror transitions, their expressions as a function of the mixing ratio $\rho$, in the limit of zero momentum transfer, are [17]

$$
a_{\beta \nu}(0)=\left(1-\rho^{2} / 3\right) /\left(1+\rho^{2}\right)
$$

and

$$
A_{\beta}(0)=\frac{\rho^{2}-2 \rho \sqrt{J(J+1)}}{\left(1+\rho^{2}\right)(J+1)}
$$

where $J$ denotes the spin of the initial and final states in the transition. At a precision level of about $1 \%$, as is the case for the correlation coefficients we are dealing with here, the impact of recoil effects have however to be considered. To first order in recoil, assuming the absence of second class currents [18] and time reversal invariance, one then has for a $\beta^{+}$transition within a common isotopic multiplet [19]

$$
a_{\beta \nu}=f_{2}(E) / f_{1}(E),
$$

and

$$
A_{\beta}=f_{4}(E) / f_{1}(E),
$$

with the spectral functions

$$
\begin{gathered}
f_{1}(E)=a^{2}+c^{2}-\frac{2 E_{0}}{3 M}\left(c^{2}-c b\right)+\frac{2 E}{3 M}\left(3 a^{2}+\right. \\
\left.+5 c^{2}-2 c b\right)-\frac{2 m_{e}^{2}}{3 E M}\left(c^{2}-c b\right), \\
f_{2}(E)=a^{2}-\frac{1}{3} c^{2}+\frac{2 E_{0}}{3 M}\left(c^{2}-c b\right)-\frac{4 E}{3 M}\left(3 c^{2}-c b\right),
\end{gathered}
$$

and

$$
\begin{aligned}
f_{4}(E)= & \left(\frac{J}{J+1}\right)^{1 / 2}\left[2 a c-\frac{2 E_{0}}{3 M}(a c-a b)+\right. \\
& \left.+\frac{2 E}{3 M}(7 a c-a b)\right]+\left(\frac{1}{J+1}\right)\left[c^{2}+\frac{2 E_{0}}{3 M}\left(-c^{2}+\right.\right. \\
& \left.+c b)+\frac{E}{3 M}\left(-11 c^{2}+5 c b\right)\right] .
\end{aligned}
$$

Here $E$ and $E_{0}$ denote respectively the total and the total maximal positron energies, $M$ is the average mass of the mother and daughter isotopes, and $m_{e}$ is the electron mass. In this notation [19] $a, b$ and $c$ designate respectively, the Fermi-, weak magnetism- and Gamow-Teller form factors

$$
a=g_{V} M_{F}, c=g_{A} M_{G T} .
$$

with $C_{i}=V_{u d} G_{F} g_{i}\left(q^{2} \rightarrow 0\right),(i=V, A), g_{i}$ being the vector and axial-vector form factors and $q$ the momentum transfer. According to the conserved-vector-current hypothesis 19, 20]

$$
b=A \sqrt{(J+1) / J} M_{F} \mu .
$$

where $A$ is the mass number and $\mu=\left[\mu\left(T_{3}\right)-\right.$ $\left.\mu\left(T_{3}^{\prime}\right)\right] /\left(T_{3}-T_{3}^{\prime}\right)$ is the isovector contribution to the magnetic moment, with $T_{3}$ the third component of the isospin (in the convention where $T_{3}=+1 / 2$ for a proton) and $\mu\left(T_{3}\right)$ and $\mu\left(T_{3}^{\prime}\right)$ the magnetic moments of the mother and daughter nuclei.

The extraction of $\left|V_{u d}\right|$ proceeds then by solving Eqs.(10) or (11) for $\rho$ and then inserting its value in Eq.(6) with the corresponding $\mathcal{F} t$ value from Ref. [15] yielding

$$
V_{u d}^{2}=\frac{K^{\prime}}{\mathcal{F} t\left(1+f_{A} \rho^{2} / f_{V}\right)},
$$


where $K^{\prime}=K /\left[G_{F}^{2} C_{V}^{2}\left(1+\Delta_{R}^{V}\right)\right]=5831.3(22) \mathrm{s}$, and $\Delta_{R}=2.361(38) \%$ [16]. The error on $K^{\prime}$ is dominated by the uncertainty on the radiative corrections $\Delta_{R}$. The sign of $\rho$ was taken to be the same as in Ref. [15].

The data included in the present analysis is summarized in Table I. The mirror transitions considered here are those in ${ }^{19} \mathrm{Ne},{ }^{21} \mathrm{Na}$ and ${ }^{35} \mathrm{Ar}$, for which the betaneutrino angular correlation coefficient or the beta decay asymmetry parameter have been measured. The inclusion of recoil effects in the $\mathcal{F} t$ values was found to have negligible effects on the resulting values for $\left|V_{u d}\right|$. Corrections for $\delta_{R}^{\prime}, \delta_{N S}$ and $\delta_{C}$ in the correlation coefficients cancel in the ratios of the spectral functions, Eqs.(10) and (11). Electromagnetic corrections [19], other than the dominant Coulomb effects contained in the energydependent Fermi function $F(Z, E)$ and included in the $f_{V, A}$ factors, were verified to be negligible at the present level of precision. The values for $E$ used in Eqs.(10) and (11) and listed in Table [ are average values determined from the experimental conditions.

\begin{tabular}{lrrr}
\hline \hline & \multicolumn{1}{c}{${ }^{19} \mathrm{Ne}$} & \multicolumn{1}{c}{${ }^{21} \mathrm{Na}$} & \multicolumn{1}{c}{$\mathrm{Ar}$} \\
\hline$a_{\beta \nu}$ & - & $0.5502(60)^{a}$ & - \\
$A_{\beta}$ & $-0.0391(14)^{b}$ & - & $0.430(22)^{c}$ \\
$\mathcal{F} t[\mathrm{~s}]^{d}$ & $1718.4(32)$ & $4085(12)$ & $5688.6(72)$ \\
$f_{A} / f_{V}{ }^{d}$ & 1.01428 & 1.01801 & 0.98938 \\
$E_{0}[\mathrm{MeV}]^{e}$ & $2.72783(30)$ & $3.03658(70)$ & $5.45514(70)$ \\
$E[\mathrm{MeV}]$ & 0.510999 & $1.614(1)$ & $2.780(1)$ \\
$M[\mathrm{amu}]^{e}$ & $19.0001417(7)$ & $20.9957509(10)$ & $34.9720551(14)$ \\
$b^{f}$ & $-148.5605(26)$ & $82.6366(27)$ & $-8.5704(90)$ \\
\multicolumn{1}{c}{} & & & \\
$\left|V_{u d}\right|$ & $0.9716(22)$ & $0.9696(36)$ & $0.9755(38)$ \\
\hline \hline
\end{tabular}

TABLE I: Input data used to determine the values of $\rho$ and $\left|V_{u d}\right|$ from the mirror transitions in ${ }^{19} \mathrm{Ne},{ }^{21} \mathrm{Na}$ and ${ }^{35} \mathrm{Ar}$.

${ }^{a}$ From Ref. 21].

${ }^{b}$ Value for $\mathrm{E}=\mathrm{m}_{e}$, from Ref. [22].

${ }^{c}$ Weighted mean of values from Refs. 23] and 24].

${ }^{d}$ From Ref. [15].

${ }^{e}$ Using data from Ref. [25].

${ }^{f}$ Calculated with the magnetic moments listed in Ref. [26].

The beta asymmetry parameter in ${ }^{19} \mathrm{Ne}$ decay has been measured a couple of decades ago by the Princeton group [22, 27]. Although the value reported in 27] has a better precision than the results quoted in [22], we do not include here that input since the result has never been published. From the value reported in 22], i.e. $A_{\beta}=-0.0391(14)$, the value $\rho=1.5995(46)$ is extracted, leading to $\left|V_{u d}\right|\left({ }^{19} \mathrm{Ne}\right)=0.9716(22)$.

A recent measurement of the beta-neutrino angular correlation coefficient in ${ }^{21} \mathrm{Na}$ decay produced the value $a_{\beta \nu}=0.5502(60)$ 21]. This result constitutes the most precise measurement of this coefficient in a mirror transition. The value of the mixing ratio extracted from this result is $\rho=-0.7136(72)$ leading to $\left|V_{u d}\right|\left({ }^{21} \mathrm{Na}\right)=$ 0.9696(36).

Finally, in the decay of ${ }^{35} \mathrm{Ar}$, the beta asymmetry parameter has reliably been measured twice, with the results $A_{\beta}=0.49(10)$ [23] and $A_{\beta}=0.427(23)$ 24]. The weighted mean of these (Table \), which is dominated by the most precise of both, yields a value $\rho=-0.279(15)$, leading to $\left|V_{u d}\right|\left({ }^{35} \mathrm{Ar}\right)=0.9755(38)$.

Except for ${ }^{19} \mathrm{Ne}$, the recoil corrections appeared not to have a significant impact in the determination of $\rho$. For ${ }^{19} \mathrm{Ne}$, Eq. (9) yields $\rho=1.6015(44)$ what differs by about half a standard deviation from the value quoted above. For ${ }^{21} \mathrm{Na}$ and ${ }^{35} \mathrm{Ar}$ the values of $\rho$ obtained from Eqs. (8) and (9) are identical to those given above. The values of $\rho$ and $\left|V_{u d}\right|$ are also summarized in Table \. The results obtained from ${ }^{21} \mathrm{Na}$ and ${ }^{35} \mathrm{Ar}$ have comparable uncertainties, which are a factor of 1.7 larger than the uncertainty on the value obtained from ${ }^{19} \mathrm{Ne}$. The weighted mean of the three values is

$$
\left|V_{u d}\right|=0.9719(17) \text { (nuclear mirror transitions) }
$$

This result is consistent within 1.2 combined standard deviations with the value obtained from nuclear superallowed $0^{+} \rightarrow 0^{+}$transitions, Eq.(2), and has an uncertainty comparable to that obtained from neutron decay, Eq.(3). This shows that nuclear mirror transitions provide an independent sensitive source for the determination of $\left|V_{u d}\right|$ and deserve therefore further theoretical studies and experimental investigations to improve the required inputs.

The survey in Ref. 15] reports, for each decay, the contributions of the five inputs $\left(f_{V}\right.$, lifetime, branching ratio, $\delta_{R}$ and $\left.\delta_{C}-\delta_{N S}\right)$ to the error on the $\mathcal{F} t$ values. For ${ }^{19} \mathrm{Ne}$ the uncertainty is dominated by experimental inputs to determine $f_{V}$ and by the lifetime. The situation is similar in ${ }^{21} \mathrm{Na}$ where in addition, the uncertainty on the branching ratio contributes at the third place. Except for ${ }^{35} \mathrm{Ar}$, where all five inputs are known with a relative uncertainty below $10^{-3}$, all other transitions ranging from ${ }^{3} \mathrm{He}$ to ${ }^{45} \mathrm{~V}$ have uncertainties dominated by the experimental inputs. Improvements in the determination of $\left|V_{u d}\right|$ offer therefore new opportunities for precision experiments in mirror transitions. However, the uncertainties on the values of $\left|V_{u d}\right|$ given in Table $\Pi$ are dominated by those on $\rho$, so that improvements of these values call in priority for new measurements of correlation coefficients.

We consider here in particular the impact of a new measurement of $a_{\beta \nu}$ in ${ }^{19} \mathrm{Ne}$ and ${ }^{35} \mathrm{Ar}$ with a similar precision to that achieved for ${ }^{21} \mathrm{Na}$ [21]. For this purpose we use Eqs.(8) and (9) instead of Eqs.(10) and (11).

Figure 1 shows the sensitivity of $a_{\beta \nu}$ and $A_{\beta}$ to $\rho$ for ${ }^{19} \mathrm{Ne}$ decay. The solid lines are the differences between Eq.(9) and the experimental values (Table I) at $\pm 1 \sigma$. The dotted lines are the differences between Eq. (8) and the values of $a_{\beta \nu}$ calculated with Eq.(8) using the value of $\rho$ given in Table $\square$ and assuming a relative precision 
of $\pm 1 \%$ on $a_{\beta \nu}$. The two solid and the two dotted lines are superimposed on the left panel. The right panel in Fig 1 shows a zoom to the intersection region of the two curves with zero. It is seen that a measurement of $a_{\beta \nu}$ for ${ }^{19} \mathrm{Ne}$ with a $1 \%$ relative uncertainty enables to reduce the uncertainty on $\rho$ by a factor of about 3. The sensitivities of the two observables to $\rho$ are comparable.
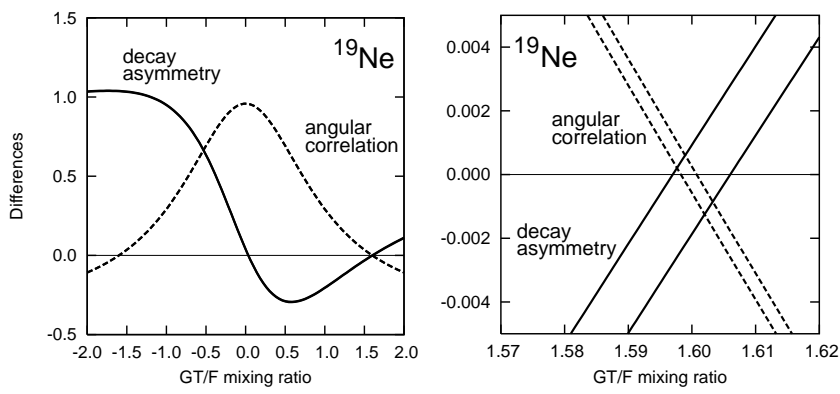

FIG. 1: Left panel: sensitivity of the angular correlation coefficient and of the decay asymmetry parameter to the mixing ratio in ${ }^{19} \mathrm{Ne}$ decay. Right panel: intersection between the two curves. The dotted lines indicate the region allowed by a measurement of $a_{\beta \nu}$ with a relative uncertainty of $\pm 1 \%$.

Figure 2 shows the same analysis for ${ }^{35} \mathrm{Ar}$ decay. The improvement is here very moderate since the intersection of the two curves with zero occurs in the region where the sensitivity of $A_{\beta}$ to $\rho$ is the largest. In fact, among all mirror transitions considered in Ref. [15], it appears that $a_{\beta \nu}$ shows the largest sensitivity to $\rho$ in ${ }^{19} \mathrm{Ne}$ decay and the smallest sensitivity in ${ }^{35} \mathrm{Ar}$.
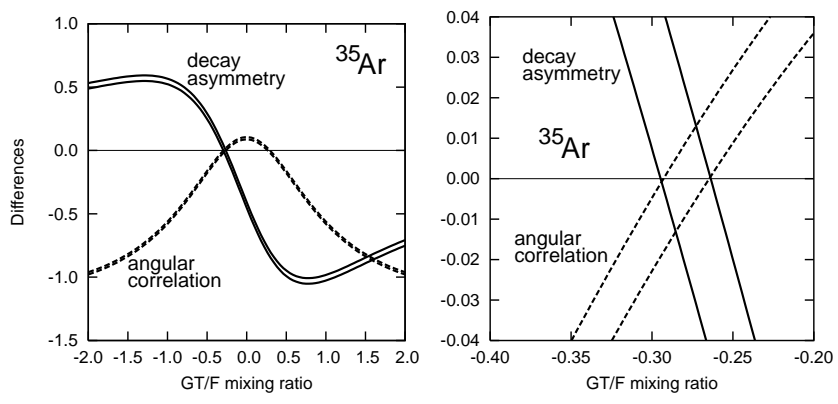

FIG. 2: Same than Fig. 1 but for ${ }^{35}$ Ar decay.

Based on the values of $\mathcal{F} t$ and $\rho$ listed in Ref. [15] we found that measurements of $a_{\beta \nu}$ provide better prospects than $A_{\beta}$ to improve on the value of $\left|V_{u d}\right|$ from mirror transitions, the highest sensitivities being obtained for ${ }^{3} \mathrm{He},{ }^{17} \mathrm{~F},{ }^{19} \mathrm{Ne}$ and ${ }^{41} \mathrm{Sc}$. In the same context, measurements of $A_{\beta}$ look of interest only in ${ }^{19} \mathrm{Ne}$ decay, the sensitivity being then similar to that of $a_{\beta \nu}$ in the same decay, as shown above.

In conclusion, we have deduced the value of the CKM matrix element $\left|V_{u d}\right|=0.9719 \pm 0.017$ using only data from transitions in ${ }^{19} \mathrm{Ne},{ }^{21} \mathrm{Na}$ and ${ }^{35} \mathrm{Ar}$. This demonstrates that nuclear mirror transitions provide an independent sensitive source for the determination of $\left|V_{u d}\right|$. Further theoretical studies as well as precise determinations of the experimental inputs, and in particular of the correlation coefficients, are desirable.

[1] N. Cabibbo, Phys. Rev. Lett. 10, 531 (1963).

[2] M. Kobayashi and T. Maskawa, Prog. Theor. Phys. 49, 652 (1973).

[3] I.S. Towner and J.C. Hardy, arXiv:nucl-th/9809087v1, and in Proc. of the $5^{\text {th }}$ Int. WEIN Symp. Physics Beyond the Standard Model, P. Herczeg, C.M. Hoffman and H.V. Klapdor eds. (World Scientific, Singapore, 1999).

[4] I.S. Towner and J.C. Hardy J. Phys. G: Nucl. Part. Phys. 29, 197 (2003).

[5] J.C. Hardy, arXiv:hep-ph/0703165 1.

[6] J.C. Hardy and I.S. Towner, Phys. Rev. C 71, 055501 (2005).

[7] I.S. Towner and J.C. Hardy, Phys. Rev. C 77, 025501 (2008).

[8] D. Thompson, J. Phys. G: Nucl. Part. Phys. 16, 1423 (1990).

[9] C. Amsler et al. (Particle Data Group), Phys. Lett. B 667, 1 (2008).

[10] J.S. Nico and W.M. Snow, Annu. Rev. Nucl. Part. Sci. 55, 27 (2005).

[11] H. Abele, Prog. Part. Nucl. Phys. 60, 1 (2008) and references therein.

[12] H. Abele, et al. Phys. Rev. Lett. 88, 211801 (2002).

[13] See contributions to Proc. Int. Workshop on Particle Physics with Slow Neutrons, Grenoble May 2008, to appear in Nucl. Instr. and Meth. in Phys. Res. A.

[14] D. Pocanic et al., Phys. Rev. Lett. 93, 181803 (2004).

[15] N. Severijns, M. Tandecki, T. Phalet and I.S. Towner, arXiv:nucl-ex/08072201v1, submitted to Phys. Rev. C.

[16] W.J. Marciano and A. Sirlin, Phys. Rev. Lett. 96, 032002 (2006).

[17] See e.g. N. Severijns, M. Beck and O. Naviliat-Cuncic, Rev. Mod. Phys. 78, 991 (2006).

[18] For a review see L. Grenacs, Annu. Rev. Nucl. Part. Sci. 35, 455 (1985).

[19] B.R. Holstein, Rev. Mod. Phys. 46, 789 (1974) and 48, 653 (1976).

[20] B.R. Holstein and S.B. Treiman, Phys. Rev. 3, 1921 (1971).

[21] P.A. Vetter, J.R. Abo-Shaeer, S.J. Freedman and R. Maruyama, Phys. Rev. C 77, 035502 (2008).

[22] F.P. Calaprice, S.J. Freedman, W.C. Mead and H.C. Vantine, Phys. Rev. Lett. 35, 1566 (1975).

[23] J.D. Garnett, E.D. Commins, K.T. Lesko and E.B. Norman, Phys. Rev. Lett. 60, 499 (1988).

[24] A. Converse et al., Phys. Lett. B 304, 60 (1993).

[25] G. Audi, O. Bersillon, J. Blachot and H. Wapstra, Nucl. Phys. A 729, 3 (2003).

[26] N.J. Stone, At. Data and Nucl. Data Tabl. 90, 75 (2005).

[27] D.F. Schreiber, Ph.D. thesis, Princeton University, (1983). 